Chirurg 2014 · 85:852-853

DOI 10.1007/s00104-014-2758-2

Online publiziert: 20. September 2014

(c) Springer-Verlag Berlin Heidelberg 2014

\section{B. Bouillon ${ }^{1} \cdot H$. Windhagen ${ }^{2}$}

${ }^{1}$ Klinik für Orthopädie, Unfallchirurgie und Sporttraumatologie, Lehrstuhl der Universität Witten Herdecke am Klinikum Köln-Merheim, Köln

${ }^{2}$ Orthopädische Klinik der Medizinischen Hochschule Hannover,

Im Diakoniekrankenhaus Annastift gGmbH, Hannover

\title{
Verletzungen bei Sportlern
}

\section{Neues zu Diagnostik und Therapie häufiger Sportverletzungen}

Der Sport spielt in unserer Gesellschaft eine große Rolle. Dies gilt für den Leistungssport genauso wie für den Breitensport. In Deutschland sind 27\% der Menschen Mitglied in einem Sportverein [2]. Der gesundheitliche Nutzen des Sports ist für definierte Erkrankungen wie z. B. die Reduktion von Herz-Kreislauf-Erkrankungen belegt [5]. Darüber hinaus kann Sport im Sinne der Sekundärprävention eine medikamentöse Therapie bei Volkskrankheiten wie der koronaren Herzkrankheit, dem Schlaganfall, der Herzinsuffizienz, dem Diabetes mellitus und der Arthrose zumindest teilweise ergänzen oder gar ersetzen [6]. Dem gesundheitlichen Mehrwert des Sporttreibens steht jedoch das Verletzungsrisiko gegenüber. In Deutschland ist jährlich mit ca. 2 Mio. Sportverletzungen zu rechnen [4].

Verletzungen im Sport lassen für viele insbesondere ambitionierte Sportler eine „Welt zusammenbrechen“. Sportler wollen im Fall einer Verletzung die optimale Beratung, die sofortige umfassende Diagnostik und die beste verfügbare Therapie bezogen auf ihre ganz persönlichen Bedürfnisse und privaten wie beruflichen Lebensumstände. Für viele Sportler sind die wichtigsten Fragen, wo sie den besten Sportmediziner finden, wie lange sie ausfallen und ob sie kompetitiv ihren Sport wieder ausüben können.

Doch am Anfang steht erst einmal eine gute und dem wissenschaftlichen Stand des Wissens entsprechende Beratung. Daher ist das Ziel dieser Ausgabe des Chi- rurgen, anlässlich des Deutschen Kongresses für Orthopädie und Unfallchirurgie (DKOU 2014) Neues zu Diagnostik und Therapie häufiger Sportverletzungen bzw. Überlastungsschäden so darzustellen, dass Sie in die Lage versetzt werden, Ihre Sportler bestmöglich zu beraten bzw. zu behandeln. Der DKOU 2014 steht unter dem Motto „Wissen schafft Vertrauen“. In diesem Sinn haben wir Experten gebeten, neue Entwicklungen in Bezug auf Diagnostik und Therapie häufiger Sportverletzungen aufzubereiten und zu diskutieren.

\section{) Die Evidenz in der Behandlung von Sportverletzungen muss verbessert werden}

Die Sportverletzungen, die wir diskutieren möchten, sind die Schultereckgelenksverletzung, die Schulterluxation, das femoroazetabuläre Impingement, die patellofemorale Instabilität und die vordere Kreuzbandverletzung. Themen, die im Sinne eines Update thematisiert werden, sind diagnostische Modalitäten, Therapieoptionen, Kriterien zur Individualisierung und Entscheidungsfindung der Therapie, Zeitpunkt einer möglichen operativen Versorgung, konservative und operative Techniken, Aspekte der Nachbehandlung und Rehabilitation, Behandlungsergebnisse und Zeitpunkt der Wiederaufnahme der sportlichen Aktivitäten.
Die Evidenzlage bei der Versorgung von Sportverletzungen ist sehr unterschiedlich. Insbesondere klinische relevante Fragen sind häufig nicht adäquat adressiert. Es fehlen hochwertige Studien mit großen Patientenzahlen selbst bei häufigen Verletzungen. Eine Stratifizierung der untersuchten Populationen nach Aktivitätslevel und Sportart findet man nur selten.

Im onkologischen Bereich der Medizin existiert seitens der Erwartungshaltung der Patienten durchaus ein Bewusstsein für die Notwendigkeit randomisierter Studien. Bei Sportverletzungen ist dies "gefühlt“ anders. Der Sportler möchte nichts dem Zufall überlassen und schon gar nicht eine Therapieentscheidung. Dies gilt umso mehr, wenn es um die Entscheidung zwischen einer konservativen oder operativen Therapie geht.

Stellen Sie sich vor, dass der Mannschaftsarzt einer 1.-Bundesliga-Fußballmannschaft dem verletzten Spieler erklärt, dass wissenschaftlich nicht klar ist, ob eine operative Versorgung seiner Schultereckgelenksverletzung Typ Rockwood III notwendig ist oder nicht... und er daher den Spieler fragt, ob er bereit ist, an einer Studie teilzunehmen, bei der ein Briefumschlag entscheidet, ob er operativ versorgt wird oder nicht. Wir möchten hier nicht ein Plädoyer gegen die Durchführung von Studien halten. Wir möchten vielmehr alle Beteiligten bitten, nachzudenken, wie wir mehr Evidenz in der Behandlung von Sportverletzungen schaffen 
können. Möglicherweise sind die skandinavischen Kreuzbandregister ein gutes Beispiel dafür, wie man das, was jeden Tag gemacht wird, so systematisch beobachtet und dokumentiert, dass ein Erkenntnisgewinn daraus gewonnen werden kann [1,3].

Wir wünsche Ihnen viel Spaß bei der Lektüre dieses Schwerpunktheftes und hoffen, Ihnen einen aktuellen Überblick über den derzeitigen Stand der Versorgung einiger häufiger Sportverletzungen geben zu können.

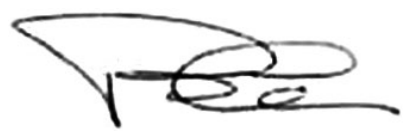

Prof. Dr. Bertil Bouillon

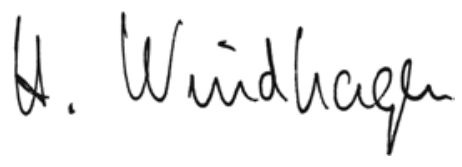

Prof. Dr. Henning Windhagen

\section{Korrespondenzadressen}

\section{Prof. Dr. B. Bouillon}

Klinik für Orthopädie, Unfallchirurgie und Sporttraumatologie, Lehrstuhl der Universität Witten Herdecke am Klinikum Köln-Merheim, Ostmerheimerstr 200, 51109 Köln

bouillonb@kliniken-koeln.de

\section{Prof. Dr. H. Windhagen}

Orthopädische Klinik der Medizinischen Hochschule Hannover, Im Diakoniekrankenhaus Annastift gGmbH,

Anna-von-Borries-Str. 1-7, 30625 Hannover Henning.Windhagen@ddh-gruppe.de

Interessenkonflikt. B. Bouillon und H. Windhagen geben an, dass kein Interessenkonflikt besteht.

\section{Literatur}

1. Andernord D, Björnsson $\mathrm{H}$, Petzold $M$ et al (2014) Surgical predictors of early revision surgery after anterior cruciate ligament reconstruction: Results from the Swedish National Knee Ligament Register on 13,102 patients. Am J Sports Med 42: 1574 1582
2. Deutscher Olympischer Sportbund (2012) DOSB Bestandserhebung 2012, Fassung vom 15.11.2012. http://www.dosb.de/de/medien/downloads/statistiken/

3. Granan LP, Forssblad M, Lind M, Engebretsen $L$ (2009) The Scandinavian ACL registries 20042007: baseline epidemiology. Acta Orthop 80: 563-567

4. Henke T, Luig P, Schulz D (2014) Sportunfälle im Vereinssport in Deutschland. Bundesgesundheitsbl 57: 628-637

5. Löllgen $H$ (2013) Bedeutung und Evidenz der körperlichen Aktivität zur Prävention und Therapie von Erkrankungen. Dtsch Med Wochenschr 138: 2253-2259

6. Naci H, lonnidis JPA (2013) Comparative effectiveness of exercise and drug interventions on mortality outcomes: a metaepidemiological study. BMJ 347:f5577

\section{Springer Medizin}

\section{Videobeitrag: Aktuelle Operationstechniken}

\section{Reichen Sie Ihren Beitrag inkl. Video für Der Chirurg jetzt ein!}

Im Mittelpunkt des Beitrags steht Ihr Video zu aktuellen Operationstechniken. Es werden bewährte operative Verfahren dargestellt. Neben der Vermittlung aller wichtigen prä-, intraund postoperativen Details sowie den Hinweisen auf mögliche

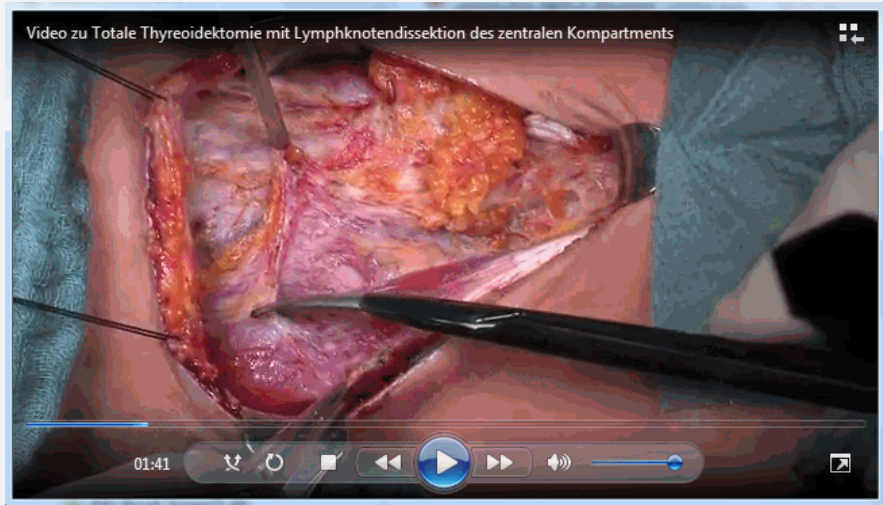

Alle Operationsschritte werden übersichtlich im Detail von Experten erklärt. Die neue Videoreihe finden Sie auf springermedizin.de
Fehler und Gefahren liegt der Schwerpunkt auf einer genauen Darstellung der jeweiligen Operationsschritte im Video.

Nutzen Sie das Medium des Videos um komplizierte Vorgehensweisen leicht verständlich zu präsentieren. Bitte reichen Sie zudem einen kurzen begleitenden Beitrag ein, der die wesentlichen Punkte des Videos zusammenfasst.

Weitere Informationenzur Einreichung sowie einen detaillierten Autorenleitfaden finden Sie auf www.derchirurg.de

Reichen Sie Ihren Videobeitrag online ein unter www.editorialmanager.com/dech/ 